\section{Prävalenzplateau in der Schweiz}

\author{
Nahezu alle westlichen Länder verzeichnen seit Jahrzehnten eine \\ stetige Zunahme von Asthma und allergischer Rhinitis bei Kindern \\ und Jugendlichen. Eidgenössische Epidemiologen konnten jetzt \\ eine Stabilisierung der Prävalenzzahlen in der Schweiz auf dem \\ Niveau des Jahres 1994 dokumentieren.
}

E in interdisziplinäres Team befragte mithilfe von Videointerviews oder klassischen Erhebungsbögen 13 bis 14 Jahre alte Schweizer mit deren Eltern zu Asthma- und Allergiesymptomen. Außerdem wurden potenzielle Risikofaktoren wie elterliche Allergie, Bildungsgrad und Haustierhaltung erfasst. Die Erhebung erfolgte in drei Zeiträumen: An der ersten Erhebung in den Jahren 1992-1993 nahmen 1.324, an der zweiten von 1995-1997 1.668 und an der dritten von 1999-2000 1.250 Jugendliche teil. Die Schüler lebten in vier ausgewählten Städten oder kamen aus vier ländlichen Regionen der Schweiz. Zusätzlich zu den Fragen wurden von einigen Teilnehmern Blutproben auf spezifische IgE-Antikörper untersucht.
Die Prävalenzrate von allergischem Asthma lag zu allen drei Befragungszeitpunkten um die 10\%. Auch bei allergischer Rhinitis war keine Zunahme zu beobachten: $16,5 \% \quad$ (1993), $13,9 \%$ (1997) und $16,1 \%$ (2000). Wie fast zu erwarten, unterschieden sich auch die Häufigkeiten von Sensibilisierungen gegen klassische Aeroallergene kaum zwischen den drei Erhebungszeiträumen. Der Einfluss der abgefragten Risikofaktoren erwies sich als minimal.

Die Autoren bieten als eine mögliche Erklärung für Ihre Beobachtung an, dass der genetische Pool, der aufgrund von Umwelteinflüssen eine atopische Erkrankung entwickeln kann, inzwischen ausgeschöpft ist. Dazu passt, dass aktuelle Studien aus Italien, den USA und der Türkei ebenfalls über stagnie-

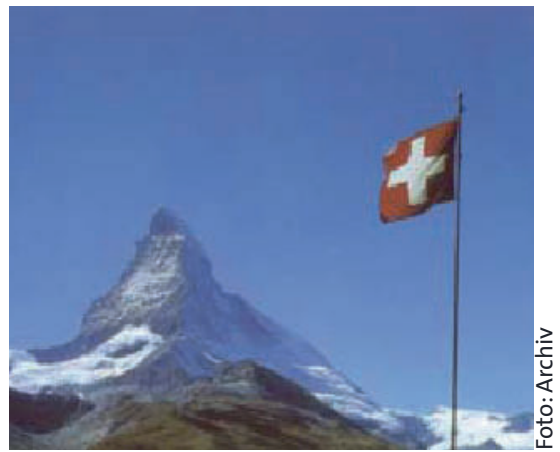

Die Spitze der Allergie- und Asthmahäufigkeit in der Schweiz scheint erreicht zu sein.

rende Prävalenzzalen berichten. Ungebrochen scheint der Trend nach oben dagegen in Australien und England.

Fazit: Die Häufigkeit von Asthma und Allergie bei jugendlichen Schweizern steigt seit Mitte der 90er Jahre nicht weiter an. Der dokumentierte Zeitraum ist allerdings noch zu kurz, um eine statistisch zuverlässige Aussage treffen zu können

mha

Braun-Fahrländer C et al. No further increase in asthma, hay fever and atopic sensitisation in adolescents living in Switzerland. Eur Respir J 2004; 23: 407-13

\title{
Zusammen leben, zusammen leiden?
}

\section{Allergische Rhinitis entsteht im Zusammenspiel von genetischen Faktoren und Umwelteinflüssen. Eine Lübecker Arbeitsgruppe un- tersuchte jetzt, welche Wirkung es hat, mit einem an Heuschnupfen erkrankten Partner zu leben - mit erstaunlichem Ergebnis.}

E pidemiologen baten über 6.000 Bürger der schwäbischen Stadt Augsburg an einer standardisierten Datenerhebung teilzunehmen. Immerhin 4.261 davon erklärten sich dazu bereit. Trainierte Interviewer arbeiteten in persönlichen Gesprächen einen Fragenkatalog zu Heuschnupfenanamnese, Ernährung, sozioökonomischem Status, Nikotinkonsum und zu den Lebensumständen ab.

Immerhin ein Fünftel aller Befragten gab an, schon einmal unter Heuschnupfen gelitten zu haben. Das Alter bei Beginn der Erkrankung lag im Mittel bei 26 Jahren. Interessant ist auch, dass Personen mit 8-jähriger Schulbildung eine Prävalenz von 9,6\% gegenüber 19,2\% nach 13-jähriger Schulzeit aufwiesen ( $p<0,0001)$.

Zum Zeitpunkt der Untersuchung lebten $76,9 \%$ aller Befragten mit einem Partner im selben Haus oder derselben Wohnung zusammen, im Mittel seit 23 Jahren. Nach statistischer Korrektur um die Faktoren Alter, Geschlecht, ernährungsbedingte Prädisposition und Schulbildung hatten diejenigen, die mit einem an Rhinitis erkrankten Partner zusammenlebten, ein doppelt so hohes Risiko (Odds Ratio [OR] 2,41), auch Heuschnupfen zu bekommen. Zudem stieg das Risiko abhängig von der gemeinsam verbrachten Zeit an (1-11 Jahre: OR 1,0; 12-23 Jahre: OR 1,8; 24-35 Jahre: OR 7,4; 36-54 Jahre: OR 13,7).

Die Ergebnisse zeigen wohl den Einfluss von - in der Partnerschaft geteilten - Umweltfaktoren in der Rhinitisgenese. Eine sehr gewagte Нypothese geht auch von einer möglichen Übertragbarkeit der Allergie aus.

Fazit: Lebenspartner von Patienten mit allergischer Rhinitis haben ein erhöhtes Risiko, selbst an Heuschnupfen zu erkranken

Schäfer T et al. Does my partner cause my allergy? Allergy 2004, 59: 781-5 